'Sección de Medicina Interna, Hospital Clínico Universidad de Chile. Santiago, Chile. ${ }^{2}$ Departamento de Imagenología, Hospital Clínico Universidad de Chile. Santiago, Chile. ${ }^{3}$ Departamento de Cardiología, Hospital Clínico Universidad de Chile. Santiago, Chile. aphDc.

Trabajo no recibió financiamiento. Los autores declaran no tener conflictos de interés.

Recibido el 10 de julio de 2020 aceptado el 15 de octubre de 2020

Correspondencia a: Dr. Alfredo Parra Lucares Sección Medicina Interna, Hospital Clínico Universidad de Chile. Santos Dumont 999, Independencia. Santiago, Chile. alfredop@ug.uchile.cl

\section{Paciente de 30 años con infarto agudo al miocardio e infección por SARS-CoV-2, presentación inhabitual de COVID-19. Caso clínico}

\author{
SEBASTIÁN WEITZ-MUÑOZ ${ }^{1}$, ALFREDO PARRA-LUCARES ${ }^{1, \mathrm{a}}$, \\ FERNANDO IHL ${ }^{1}$, CRISTÓBAL RAMOS ${ }^{2}$, \\ ERNESTO CHAIGNEAU ${ }^{3}$, MARCELO LLANCAQUEO ${ }^{3}$
}

\section{COVID-19 infection presenting as a myocardial infarction. Report of one case}

Isolated cardiac involvement of COVID-19 is an infrequent presentation, and myocardial infarction is even less common. We report a 30-year-old man presenting with retrosternal pain of insidious onset whose intensity increases suddenly. On admission, the patient had tachycardia and an EKG showed a $1 \mathrm{~mm}$ ST-elevation and diffuse PQ segment depression. Troponin was 26.9 $\mathrm{ng} / \mathrm{ml}$ (normal value $[\mathrm{NV}]<0.03$ ), inflammatory parameters were elevated, and SARS-CoV 2 PCR was positive. He was hospitalized with the diagnosis of myopericarditis secondary to SARS-CoV 2. He progressed favorably without pain during the hospital stay and with decreasing troponin values. A Cardiac Magnetic Resonance Imaging (MRI) was compatible with an infero-lateral transmural infarction. A coronary angiography showed a distal occlusion of the circumflex artery. Consequently, anticoagulation and double platelet anti-aggregation were started. The patient evolved favorably, with a decreasing troponin curve (last at discharge $0.49 \mathrm{ng} / \mathrm{ml}$ ) and a control EKG with pathological $Q$ in DIII and AvF, and symmetrically inverted T in DII, DIII, AvF, V4, V5, and V6.

(Rev Med Chile 2020; 148: 1848-1854)

Key words: Cardiovascular Diseases; Magnetic Resonance Imaging; Myocardial Infarction; Pandemics; COVID-19.
$\mathrm{E}$ n Wuhan, China, anunciaron los primeros casos de coronavirus el 31 de diciembre de 2019, y su rápida diseminación llevó a superar los 80.000 contagios $^{1}$. En una economía globalizada que conlleva, entre otras, gran desplazamiento de personas, llevó a la rápida diseminación al resto del mundo. Consecuentemente, la Organización Mundial de la Salud (OMS) el 11 de marzo de 2020 declara la enfermedad por coronavirus 2019 (COVID-19) una Pandemia. Actualmente hay más de 11 millones de casos reportados con una mortalidad global de 4,67\% ${ }^{2}$.

El virus del síndrome respiratorio agudo grave tipo 2 (SARS-CoV-2), agente causal de Covid-19, afecta principalmente al sistema respiratorio. Las manifestaciones varían desde asintomáticos (1\%), moderadas $(81,4 \%)$, severas $(13,9 \%)$ y críticas $(4,7 \%)^{3}$. Dentro de los síntomas más frecuentes están la tos $(79,4 \%)$, fiebre $(77,1 \%)$, disnea $(56,5 \%)$, mialgias $(23,8 \%)$, diarrea $(23,7 \%)$, y náuseas y vómitos $(19,1 \%)^{4}$. Hasta el momento faltan estudios locales. La principal causa de muerte es la falla respiratoria, pero el compromiso cardiovascular podría contribuir en el desenlace fatal e incluso ser el evento causal ${ }^{5}$. La injuria cardíaca (alza de troponinas sobre el percentil 99 del valor de re- 
ferencia superior) se reporta hasta en $14 \%$ de los pacientes hospitalizados ${ }^{6}$.

El virus ingresa a los neumocitos mediante la unión spike-protein con el receptor de la Enzima Convertidora de Angiotensina II (ACE II) en la membrana celular ${ }^{8}$. Posteriormente utiliza la maquinaria de esta para replicarse, induciendo daño secundario a inflamación, fibrosis y disfunción endotelial ${ }^{8,11}$. Los casos más graves desarrollan una respuesta inflamatoria sistémica, siendo referida como tormenta de citokinas ${ }^{11}$. En conjunto, se ha descrito un estado pro-coagulante de inmunotrombosis o tromboinflamación ${ }^{11}$. Además disminuye la actividad de ACE II, existiendo mayores niveles de Angiotensina II, induciendo mayor vasoconstricción y coagulación ${ }^{8,11}$.

Desde el pulmón, el virus y mediadores inflamatorios migrarían hacia el corazón, induciendo injuria cardiaca. También el estado inflamatorio puede gatillar la ruptura de placa ateromatosa y evento isquémico consecuente. Secundario a la insuficiencia respiratoria e hipoxemia, se produciría un desequilibrio entre la oferta y demanda de oxígeno en el miocardio, produciendo un infarto tipo $\mathrm{II}^{7,8,12}$.

El receptor ACE II se expresa a nivel pulmonar, renal, intestinal, cardiaco y endotelial, lo que podría explicar un daño directo en dichos órganos. Incluso, se ha demostrado la presencia del virus a nivel endotelial con inflamación difusa y disfunción endotelial ${ }^{9,10}$.

Presentamos el caso de un joven, sin comorbilidades, que ingresó por dolor torácico e infección por SARS-CoV 2, siendo diagnosticado con un Infarto agudo al miocardio (IAM).

\section{Caso clínico}

Hombre de 30 años, Venezolano, radicado en Chile desde 2017, con antecedentes de amigdalectomía en la niñez y apendicectomía el 2015, índice de masa corporal 22,5, sin consumo de drogas y sin antecedentes familiares relevantes.

Cuatro días previos a la consulta, presentó dolor urente en región retroesternal que cedía espontáneamente en segundos. Durante la madrugada despertó por dolor súbito opresivo retroesternal, intensidad 10/10, con irradiación cervical, brazo izquierdo e interescapular, que aliviaba a la sedestación y empeoraba al decúbito, asociado a disnea de reposo, palpitaciones y sudoración. Por persistencia del dolor, con respuesta parcial a paracetamol, acudió a urgencia.

Ingresó con 6 horas de evolución del cuadro agudo, destacando frecuencia cardiaca de 119 por minuto e inquieto, sin hallazgos al examen físico. Se administró analgesia endovenosa y 500mg de Ácido Acetil Salicílico, con respuesta favorable, y se realizaron exámenes complementarios: ECG de 12 derivadas (Figura 1), que se interpretó con supradesnivel del segmento ST difuso y descenso del segmento PQ en DII, V4, V5 y V6, radiografía de tórax sin hallazgos patológicos, exámenes de sangre, destacando: Polymerase Chain Reaction SARS-CoV 2 positiva, proteína $C$ reactiva 156 $\mathrm{mg} / \mathrm{L}(\mathrm{VN}<10 \mathrm{mg} / \mathrm{dL})$, Leucocitos $16.030 \mathrm{U} / \mathrm{ul}$ (VN entre 4.000-10.000 U/ul), Lactato deshidrogenasa $750 \mathrm{UI} / \mathrm{L}$ (VN 120-246 UI/L), creatinina kinasa total 926,9 U/L (VN 55-170 UI/L) y Troponina $26,9 \mathrm{ng} / \mathrm{ml}$ (VN menor a $0,034 \mathrm{ng} / \mathrm{ml}$ ), y un Ecocardiograma trans-torácico evidenciándose: Cavidades izquierdas de tamaño normal, función sistólica global y segmentaria normal, Fracción de Eyección (FE) 55\%, Strain longitudinal -18\%, función diastólica normal. Válvulas cardiacas normofuncionantes. Cavidades derechas de tamaño normal, función sistólica ventricular derecha normal. No se logra estimar presión sistólica de arteria pulmonar. Sin derrame pericárdico.

Ingresó a Intermedio Cardiológico con el diagnóstico de Miopericarditis secundaria a SARSCoV-2, y se inició Colchicina y Ketoprofeno. En ese momento, $24 \mathrm{~h}$ desde la consulta, se describieron frotes pericárdicos, sin otros hallazgos.

Evolucionó con curva de Troponina (Figura 2) en descenso y ECG seriados interpretados con resolución del segmento ST. Al segundo día se trasladó a sala de Medicina Interna.

El sexto día de hospitalización fue evaluado por Cardiología, definiéndose mantener el tratamiento y se realizó una Resonancia Nuclear Magnética (RNM) cardiaca, describiéndose en la secuencia inversión-recuperación para realce tardío, tras la inyección de gadolinio, un realce transmural del segmento inferolateral basal y medio ventricular, comprometiendo parcialmente el segmento apical inferior; en su región central, esta lesión muestra un área hipointensa que indica obstrucción microvascular, compatible con infarto transmural. No se observaron otros focos de realce ni evidencia de miocardiopatía infiltrativa (Figura 3 ). En este 


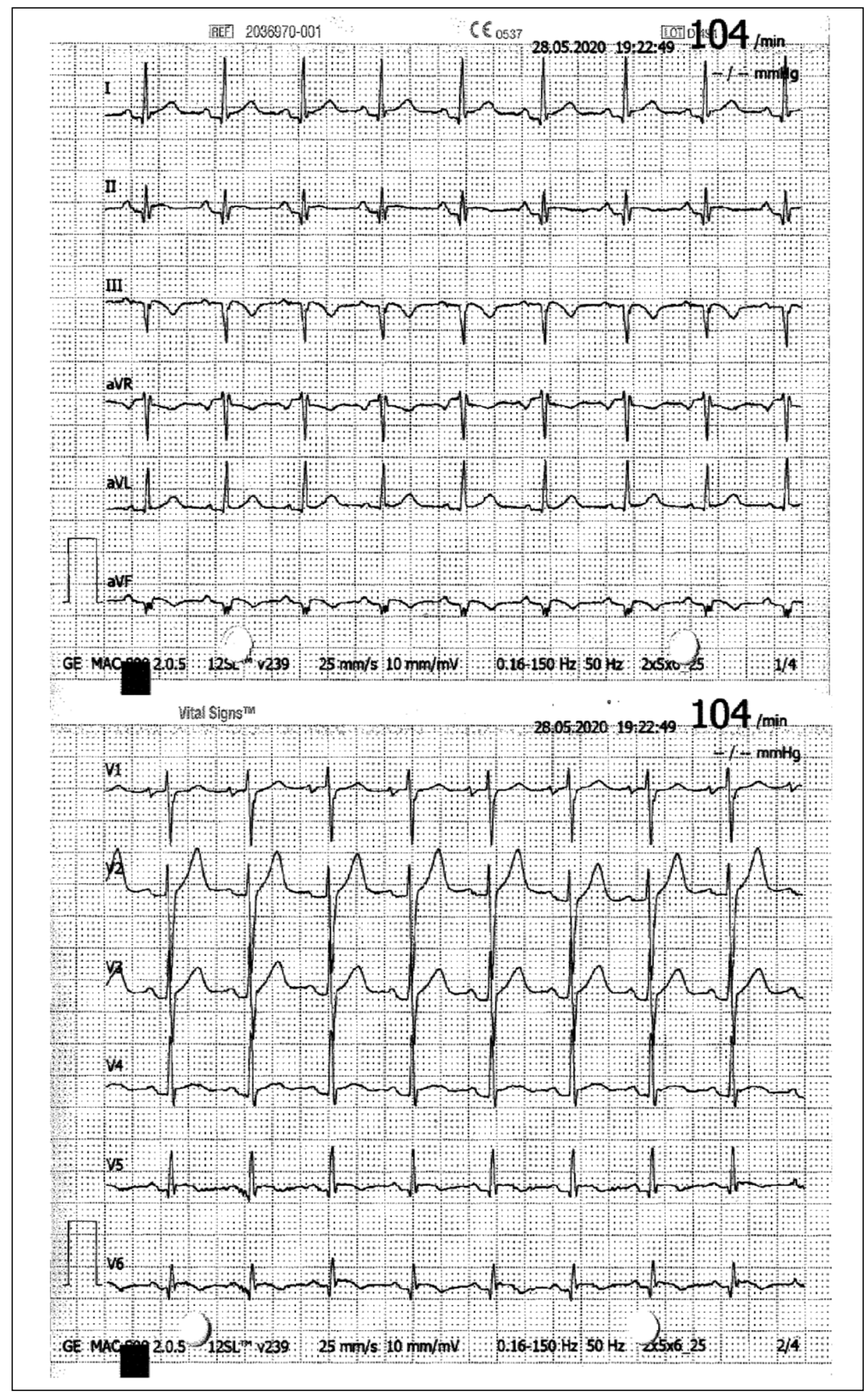

Figura 1. Electrocardiograma de 12 derivadas del ingreso. Se evidencia Taquicardia sinusal con descenso del segmento $P Q$ a nivel de DII, V4, V5 y V6, asociado a onda P negativa simétrica y ondas Q patológicas en DIII y AvF. 


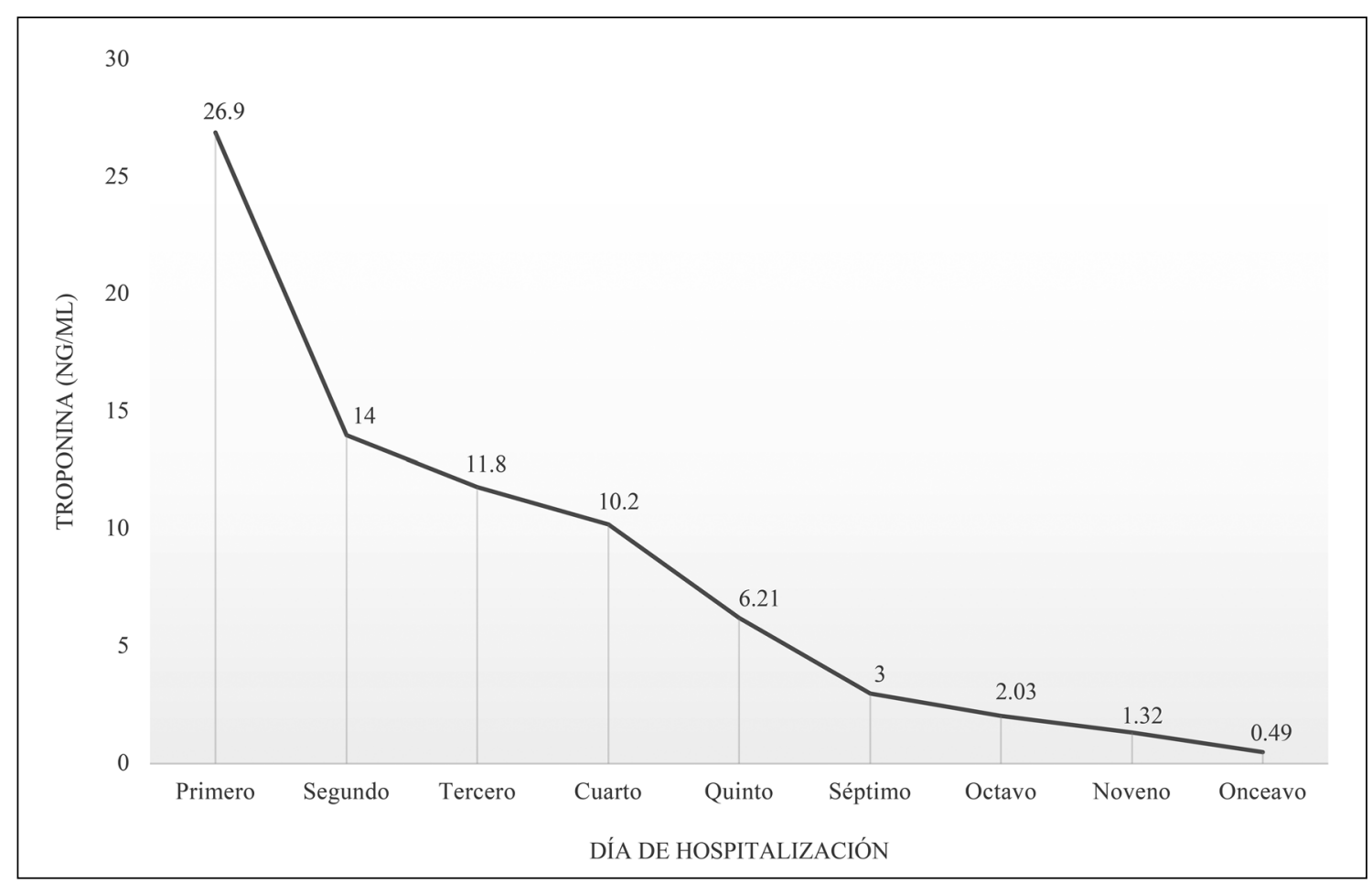

Figura 2. Curva de Troponina: A medida que van transcurriendo los días de hospitalización, los niveles de Troponina van descendiendo.
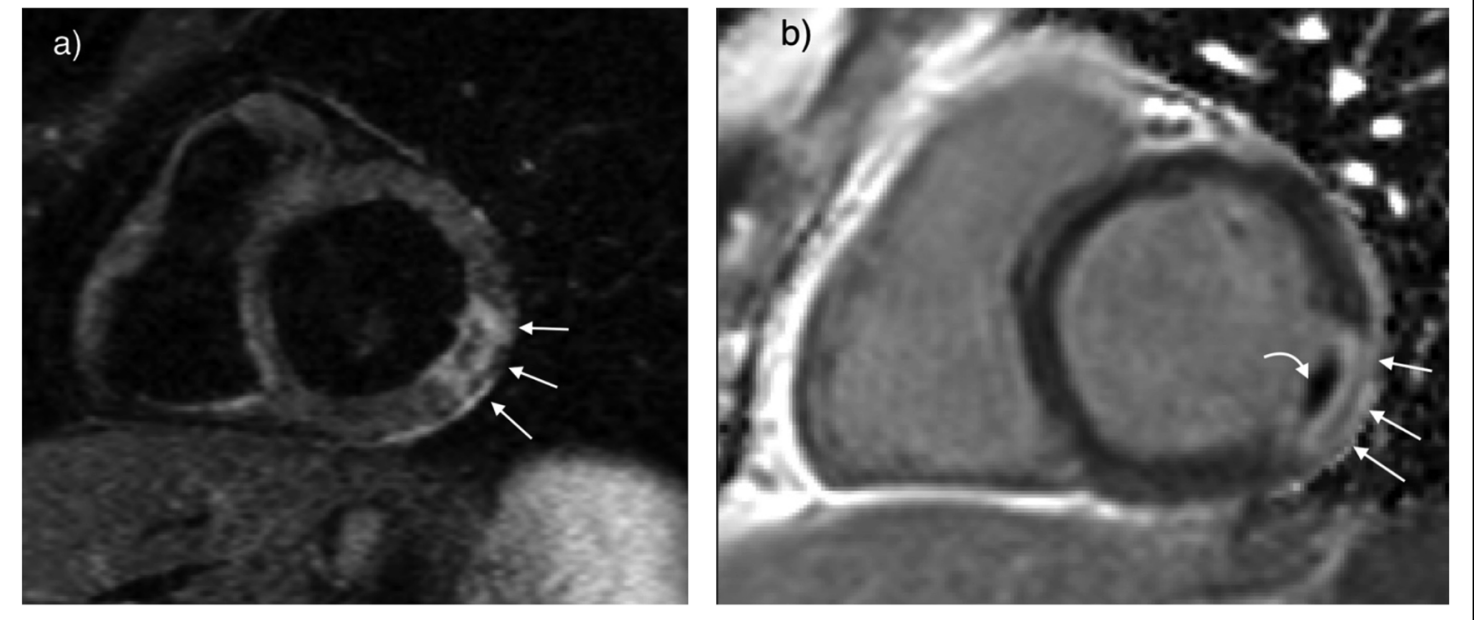

Figura 3. a) Imagen de resonancia magnética cardiaca (RMC) en eje corto secuencia $T 2$, que muestra edema transmural del segmento inferolateral basal del VI (flechas). b) Imagen de RMC en secuencia de realce tardío 10 minutos tras la inyección de gadolinio endovenoso, muestra realce transmural del segmento inferolateral basal del VI (flechas rectas), en su espesor hay una imagen hipointensa (flecha curva). Los hallazgos indican infarto agudo del miocardio con obstrucción microvascular. 


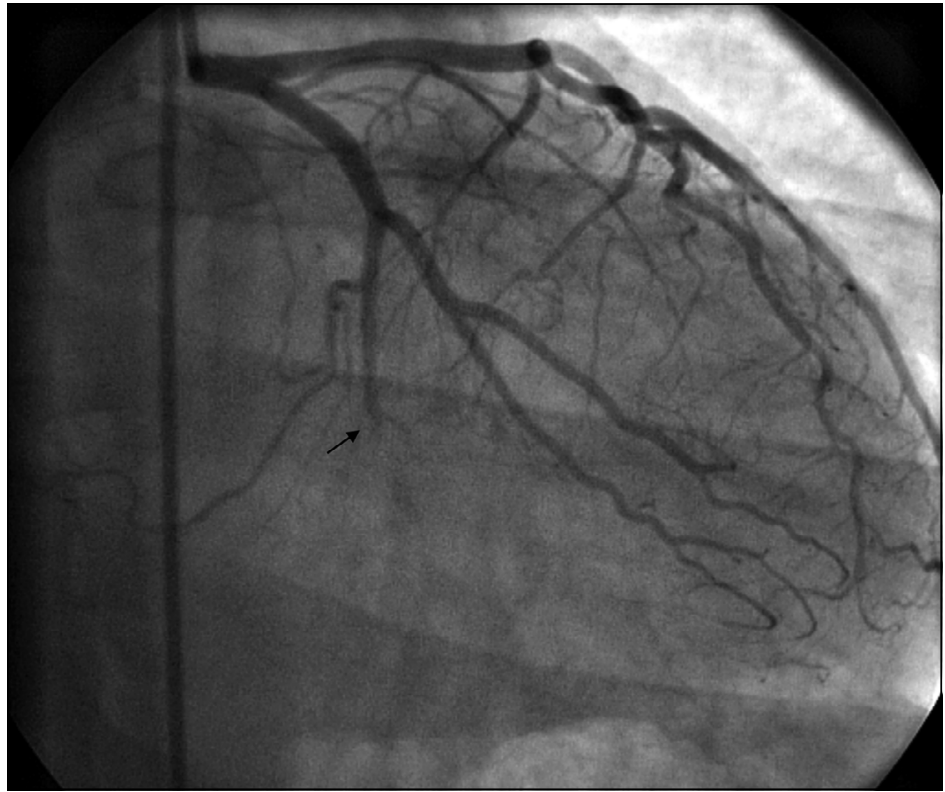

Figura 4. Coronariografía: A nivel de la flecha negra se indica la oclusión de la arteria circunfleja izquierda. contexto se realizó una Coronariografía que evidenció oclusión total distal de la arteria circunfleja y en la ventriculografía acinesia circunscrita en zona de transición postero-basal e inferior, y FE $70 \%$ (Figura 4).

Se inició tratamiento con anticoagulación, doble antiagregación plaquetaria y estatinas. Además se realizó toxicológico en orina que resultó negativo.

Paciente evolucionó sin complicaciones y descenso de Troponina hasta 0,49 ng/ml. En ECG de control con derivadas posteriores, destacó ondas T negativas y simétricas en DII, DIII, AvF, V5, V6, V7, V8 y V9, también se aprecia onda $Q$ patológica en DIII, AvF, V8 y V9, y onda U en V4.

$\mathrm{Al}$ onceavo día de hospitalización se decide alta con anticoagulación oral por 3 meses y doble antiagregación plaquetaria con estatinas por 1 año, destacando al examen físico ausencia de frotes pericárdicos.

\section{Discusión}

El COVID-19 es una infección que recién estamos conociendo. Todavía no hay claridad de los mecanismos fisiopatológicos involucrados y nueva información va surgiendo a diario. El principal órgano afectado es el pulmón y, hasta el momento, no hay una terapia conocida, siendo el tratamiento de sostén y sintomático.

Los reportes de caso de pacientes con COVID-19 e IAM son escasos. Generalmente se da en pacientes con comorbilidades asociadas, que ingresan por insuficiencia respiratoria con compromiso pulmonar, que durante la hospitalización evolucionan con IAM. Algunos reportes describen como motivo de la hospitalización al infarto, pero también son pacientes con comorbilidad asociada, síntomas respiratorios previos y/o compromiso pulmonar de por medio ${ }^{13,14}$. Los reportes de pacientes jóvenes con compromiso cardiológico, son miopericarditis, con o sin compromiso pulmonar concomitante ${ }^{15-17}$. No se encontraron reportes de pacientes jóvenes con IAM.

Nuestro caso difiere de los demás reportados, es joven, sin antecedentes mórbidos o familiares de importancia, y nunca presentó síntomas respiratorios, o de otro sistema, tampoco hubo compromiso pulmonar en la Radiografía de Tórax o al examen físico, la gasometría fue normal y nunca requirió oxigenoterapia.

En primera instancia se consideró como diagnóstico una miopericarditis, dado por edad, ausencia de factores de riesgo cardiovascular, la clínica, los hallazgos electrocardiográficos, el 
alza de parámetros inflamatorios y la ausencia de alteraciones en la motilidad segmentaria en la Ecocardiografía. Retrospectivamente, hay elementos que nos pueden hacer pensar en IAM, sin embargo, la miocarditis puede manifestarse simulando un síndrome coronario agudo, con alteraciones electrocardiográficas y elevación de troponinas similares a un IAM de pared inferior ${ }^{15,16,18}$. La RNM Cardiaca permite mejor caracterización del daño miocárdico, cada vez más usada en nuestro centro, para diferenciar con mayor precisión en los pacientes con dolor torácico y elevación de troponinas, patrones isquémicos de los no isquémicos. En nuestro caso fueron los hallazgos de la RNM Cardiaca, compatibles con una necrosis transmural, los que finalmente orientaron el diagnóstico, siendo la oclusión distal de la arteria circunfleja lo que confirma el IAM como diagnóstico definitivo.

Resulta difícil pensar que un paciente con éstas características tenga un IAM, pero considerando la inmunotrombosis mediante la activación plaquetaria, del factor tisular y la cascada de coagulación, sumado a la inhibición de anticoagulantes endógenos y fibrinolisis, se genera un estado de hipercoagulabilidad e hiperviscosidad sanguínea que favorece el desarrollo de fenómenos de microtrombosis, siendo actualmente uno de los mecanismo que explicaría en mayor medida el daño de los distintos órganos ${ }^{11}$. En conjunto, el daño endotelial también conlleva a un estado procoagulante local e induce mayor vasoconstricción. Entonces, al considerar estos factores, resulta factible pensar en fenómenos trombóticos a nivel coronario e infartos consecuentes. Dentro del diferencial destaca la embolía coronaria dados los hallazgos coronariograficos asociado al estado de hipercoagulabilidad sistémica, no tenemos documentada historia de fibrilación auricular $\mathrm{u}$ otro mecanismo embolígeno. Finalmente se han reportado fenómenos autoinmunitarios que se activan por SARS-CoV-2 como el síndrome antifosfolípido que dada su anticoagulación inmediata, post-coronariografia, imposibilita ir tras el diagnóstico en agudo ${ }^{19}$.

Seguiremos viendo casos de COVID-19 con presentaciones atípicas o fenómenos tromboembólicos de por medio, por lo cual sugerimos ampliar el campo de diagnósticos diferenciales. Grupos de expertos llaman a no olvidar al infarto en tiempos de pandemia ${ }^{18,20}$.

\section{Referencias}

1. Huang C, Wang Y, Li X, Ren L, Zhao J, Hu Y et al. Clinical features of patients infected with 2019 novel coronavirus in Wuhan, China. Lancet 2020; 395 (10239): 497-506.

2. Johns Hopkins Coronavirus Resource Center (https:// www.coronavirus.jhu.edu). Última vez revisado: 05/07/2020.

3. Wang D, Hu B, Hu C, Zhu F, Liu X, Zhang J et al. Clinical characteristics of 138 hospitalized patients with 2019 novel Coronavirus-infected Pneumonia in Wuhan, China. JAMA 2020; 323 (11): 1061-9.

4. Wu Z, McGoogan JM. Characteristics of and important lessons from the coronavirus disease 2019 (COVID-19) outbreak in China: summary of a report of 72.314 cases from the Chinese Center for Disease Control and Prevention. JAMA 2020. https://doi.org/10.1001/ jama.2020.2648.

5. Goyal P, Choi JJ, Pinheiro LC, Schenck EJ, Chen R, Jabri A, et al. Clinical characteristics of Covid-19 in New York City. N Engl J Med 2020.

6. Shi S, Qin M, Shen B, Cai Y, Liu T, Yang F, et al. Association of cardiac injury with mortality in hospitalized patients with COVID-19 in Wuhan, China. JAMA Cardiol 2020.

7. Madjid M, Safavi-Naeini P, Solomon SD, Vardeny O. Potential Effects of Coronaviruses on the Cardiovascular System: A Review. JAMA Cardiol. Published online March 27, 2020. doi:10.1001/jamacardio.2020.1286.

8. European Society of Cardiology, ESC Guidance for the Diagnosis and Management of CV Disease during the COVID-19 Pandemic, Páginas 18-20, junio 2020.

9. Ferrario CM, Jessup J, Chappell MC, Averill D, Brosnihan B, Tallant A et al. Effect of angiotensin-converting enzyme inhibition and angiotensin II receptor blockers on cardiac angiotensin-converting enzyme 2. Circulation 2005; 111 (20): 2605-10.

10. Varga Z, Flammer AJ, Steiger P, Haberecker M, Andermatt R, Zinkernagel A, et al. Endothelial cell infection and endotheliitis in COVID-19. Lancet 2020; 395 (10234): 1417-18.

11 Henry BM, Vikse J, Benoit S, Favaloro EJ, Lippi G. Hyperinflammation and derangement of renin-angiotensin-aldosterone system in COVID-19: A novel hypothesis for clinically suspected hypercoagulopathy and microvascular immunothrombosis. Clin Chim Acta. 2020;507:167-73. doi:10.1016/j.cca.2020.04.027.

12. Dhakal BP, Sweitzer NK, Indik JH, Acharya D, William P. SARS-CoV-2 Infection and Cardiovascular Disease: 
COVID-19 Heart. Heart Lung Circ 2020; S1443-9506 (20): 30271-7. doi:10.1016/j.hlc.2020.05.101.

13. Castagna F, Cerrud-Rodríguez R, Villela MA, Bortnick AE. SARS-COV-2 infection presenting as ST-elevation myocardial infarction. Catheter Cardiovasc Interv 2020; 1-4. https://doi.org/10.1002/ccd.28974.

14. Bangalore S, Sharma A, Slotwiner A, Yatskar L, Harari $\mathrm{R}$, Shah B, et al. ST-segment elevation in patients with COVID-19- a case series. N Engl J Med 2020.

15. Loghin C, Chauhan S, Lawless SM. Pseudo acute myocardial infarction in a young COVID-19 patient [published online ahead of print, 2020 Apr 27]. JACC Case Rep 2020. doi:10.1016/j.jaccas.2020.04.015.

16. Luetkens JA, Isaak A, Zimmer S, Nattermann J, Sprinkart AM, Boesecke C, et al. Diffuse Myocardial Inflammation in COVID-19 Associated Myocarditis Detected by Multiparametric Cardiac Magnetic Resonance Imaging. Circ Cardiovasc Imaging 2020; 13 (5): e010897. doi:10.1161/CIRCIMAGING.120.010897.

17. Kim IC, Kim JY, Kim HA, Han S. COVID-19-related myocarditis in a 21-year-old female patient. Eur Heart J 2020; 41 (19): 1859. doi:10.1093/eurheartj/ehaa288.

18. Bennett CE, Anavekar NS, Gulati R, Singh M, Kane GC, Sandoval Y, et al. ST-segment Elevation, Myocardial Injury, and Suspected or Confirmed COVID-19 Patients: Diagnostic and Treatment Uncertainties. Mayo Clin Proc 2020; 95 (6): 1107-11. doi:10.1016/j.mayocp.2020.04.005.

19. Zhang Y, Xiao M, Zhang S, Xia P, Cao W, et al. Coagulopathy and Antiphospholipid Antibodies in Patients with COVID-19. N Engl J Med 2020; 382:e38. doi: 10.1056/NEJMc2007575.

20. Yousefzai R, Bhimaraj A. Misdiagnosis in the COVID era: When Zebras are Everywhere, Don't Forget the Horses [published online ahead of print, 2020 Apr 27]. JACC Case Rep. 2020; doi:10.1016/j.jaccas.2020.04.018. 